\title{
RECURSOS MULTIMEDIA BILINGÜES PARA MEJORAR EL APRENDIZAJE EN ASIGNATURAS DE INGENIERÍA
}

\section{BILINGUAL MULTIMEDIA RESOURCES TO IMPROVE LEARNING IN ENGINEERING SUBJECTS}

\author{
Aurora Gil de Castro*, Eduardo Cañete Carmona, \\ Ma Salud Climent Bellido, Emilio J. Palacios García, \\ Antonio Moreno Muñoz, Alfonso Yépez \\ agil@uco.es
}

Received: 30/06/2018_ Accepted: 30/10/2018

\section{Resumen}

Estudios demuestran que la tasa de éxito de las asignaturas se fomenta si los alumnos aprecian la interdisciplinariedad entre asignaturas. Por otro lado, el uso de las nuevas tecnologías como el aprendizaje multimedia resulta esencial hoy día, más aún si se realiza en un segundo idioma. En el artículo se describen una serie de recursos multimedia bilingües que pretenden servir para mejorar el aprendizaje en asignaturas de ingeniería, permitiendo desarrollar competencias transversales y específicas de las titulaciones de grado de Ingeniería Industrial.

El uso de esta experiencia puede suponer importantes mejoras a nivel de título, ya que poner de manifiesto el nexo de unión entre varias asignaturas ayuda al aprendizaje, además de ofrecer una prospección de futuro en las diversas asignaturas del grado, lo que motivará y alentará al alumnado en promocionar a los siguientes cursos. Ver una aplicación práctica concreta en $1^{\circ}$ curso (o incluso ver en los vídeos) y saber que serán capaces de realizar el dispositivo con el que se ha realizado dicho ensayo en asignaturas posteriores, animará al alumnado y les pondrá imagen a prácticas concretas de cursos siguientes, hecho que siempre es demandado por los alumnos durante los primeros cursos.

Palabras clave: electrónica, interdisciplinar, química, sensor de conductividad

\begin{abstract}
Studies show that the success rate of the subjects is encouraged if students appreciate the interdisciplinarity between subjects. On the other hand, the use of new technologies such as multimedia learning is essential today, even more if it is done in a second language. The article describes a series of bilingual multimedia resources that aim to serve to improve learning in engineering subjects, allowing to develop transversal and specific competences of Industrial Engineering degree programs.

Important improvements can also be achieved at degree's level, since highlighting the link between various subjects helps learning, as well as offering future prospects within the subjects of the degree, which will motivate and encourage students to be promoted. Seeing a specific practical application in 1st year course and know (or even watch videos) that they will be able to make the device that will be used in later subjects, will therefore encourage the students and will put image to concrete practical lessons of following courses, which is always demanded by the students during the first courses.
\end{abstract}

Keywords: Chemical, Conductivity sensor, Electronics, Interdisciplinarity

\section{INTRODUCCIÓN}

En el contexto del Espacio Europeo de Educación Superior (EEES) ¡Error! No se encuentra el origen de la referencia.] se han establecido nuevas metodologías de enseñanza como núcleo clave de su definición y desarrollo. Se plantean nuevas metodologías de evaluación y de aprendizaje como alternativa a la clase magistral con el fin de situar al alumno como elemento activo del proceso de enseñanza-aprendizaje. Por otra parte, la formación se orienta a la adquisición de competencias, es decir, a dotar gradualmente al estudiante de las capacidades que deberá aplicar en el contexto profesional/académico propio de sus estudios para obtener resultados de forma eficiente, autónoma y flexible. Ambos aspectos forman un binomio de difícil optimización ¡Error! No se encuentra el origen de la referencia.].

Con este proyecto se desarrollan varias competencias transversales y específicas de las titulaciones de grado de Ingeniería Industrial. En concreto, este proyecto de modalidad 2 está diseñado como un programa de formación para tres profesores noveles, que imparten docencia en diferentes materias de las mismas titulaciones: Química, Fundamentos de Electrónica, y Diseño de Circuitos Electrónicos y Microelectrónicos. Este trabajo es interdisciplinar; ya que reúne conceptos de diversas disciplinas, conformando un amplio conocimiento en los futuros ingenieros que cursan los diversos grados, y dándoles una visión de conjunto de la titulación desde el primer curso. La parte química científica la ha realizado el profesor de Química, y la parte electrónica se ha considerado tanto desde el punto de vista de instrumentación electrónica, como desde el diseño y la fabricación de circuitos electrónicos, ambas desarrolladas por los profesores del Departamento de Ingeniería Electrónica. De esta manera se ha establecido acercamiento, interacción e integración entre los profesores noveles y entre todas las áreas de conocimiento implicadas.

De este proyecto se pueden beneficiar un número muy elevado de alumnos/as ya que las actividades diseñadas para las asignaturas de Química y Fundamentos de Electrónica estarán dirigidas a los alumnos de primero y segundo curso respectivamente de los grados de Ingeniería Mecánica, Eléctrica y Electrónica (150 alumnos en el caso de Ingeniería Mecánica, por ejemplo), y la de Diseño de Circuitos Electrónicos para los alumnos de $4^{\circ}$ curso de Grado en Ingeniería Electrónica. 
El limitado número de horas en el cuatrimestre, unido al elevado número de alumnos en los primeros cursos de los grados de ingeniería desemboca en la imposibilidad de realizar todas las prácticas que se desean. Sería muy recomendable realizar, o al menos mostrar al alumnado en modo webminar ciertas prácticas que, por su duración y coste de los elementos empleados no se podrían implementar a modo particular con todo el alumnado. Estudios reflejan que se fomenta el éxito en las materias implicadas si los alumnos aprecian la interdisciplinariedad entre asignaturas. El uso de las nuevas tecnologías como el aprendizaje multimedia resulta esencial ¡Error! No se encuentra el origen de la referencia.], ¡Error! No se encuentra el origen de la referencia.], más aún si se realiza en un segundo idioma, ya que conocerían terminología específica de química y electrónica, útil para su formación como ingenieros.

A nivel de título también puede suponer importantes mejoras, ya que el poner de manifiesto el nexo de unión entre varias asignaturas ayuda al aprendizaje, además de ofrecer una prospección de futuro en las diversas asignaturas del grado, lo que motivará y alentará al alumnado en promocionar a los siguientes cursos. Ver una aplicación práctica concreta en $1^{\circ}$ curso y saber (o incluso ver en los vídeos) que serán capaces de realizar el dispositivo con el que se ha realizado dicho ensayo en asignaturas posteriores, animará al alumnado y le pondrá imagen a prácticas concretas de cursos siguientes, hecho que siempre es demandado por los alumnos durante los primeros cursos. Por último, sirve para potenciar la creación de ideas de aplicaciones a desarrollar que puede ser la base de sus Trabajos Fin de Grado.

En la asignatura de Química se estudia la composición de la materia y las transformaciones de la misma. Conocer la naturaleza química de conductores, semiconductores o aislantes será de interés para las asignaturas de electrónica ya que es la base de los dispositivos electrónicos. En la asignatura de Química se utilizan sensores comerciales para determinar diferentes propiedades químicas como conductividad, temperatura, $\mathrm{pH}$, etc. Cómo funciona el sensor y sus componentes básicos se analiza en las asignaturas de Electrónica, por lo que es muy interesante que exista coordinación vertical entre los profesores que imparten estas disciplinas.

Asimismo, en la asignatura de Fundamentos de electrónica se da a conocer al alumnado los fundamentos científicos y métodos tecnológicos de la electrónica moderna o de estado sólido, los dispositivos electrónicos discretos básicos (características, gráficas y modelos funcionales), así como los bloques funcionales elementales (analógicos y digitales).

Finalmente, en la asignatura de Diseño de Circuitos Electrónicos y Microelectrónicos se pretende que el alumno adquiera los conocimientos, habilidades y destrezas, para analizar, diseñar, testar y poner en fabricación cualquier circuito electrónico, tanto analógico como digital, sobre diferentes implementaciones tecnológicas y mediante el uso de las técnicas modernas de diseño, simulación y fabricación asistida por ordenador (CAD, CAS, CAM).

Por tanto, unificando estas tres asignaturas surge el proyecto que se presenta aquí, consistente en la realización de prácticas, que cubrirán diversas fases de diseño y análisis, con las que se harán vídeos explicativos en inglés y castellano para promover la difusión de dicho contenido. El proyecto constará de 3 fases: diseño de experiencias de química y electrónica para realizarlas en el aula; grabación de vídeos de dichas prácticas; y por último, creación de una web para ubicar los recursos, tanto en castellano como en inglés.

Como antecedentes a este proyecto nos encontramos con el concedido en esta misma modalidad en el anterior curso 2016/17 titulado 'Nuevas Tecnologías Aplicadas a la Enseñanza. Interdisciplinariedad entre Asignaturas', que implicaba la química con la rama de traducción e interpretación, y que se realizó con éxito logrando los objetivos que se habían propuesto. Asimismo, en los anteriores cursos, la asignatura de Fundamentos de Electrónica se ha visto implicada en diversos proyectos de innovación docente a través del inglés como es el caso del proyecto 'Mejora de los Procesos de Aprendizaje en Asignaturas de Electrónica a Través del Inglés’ (curso 2014/15), y 'Manual en Inglés de Prácticas de Fundamentos de Electrónica’ (curso 2013/14).

Actualmente, una de las competencias considerada como clave es el dominio de una lengua extranjera. Asimismo, con estos videos se pretende reforzar otra de las competencias como la básica 5 (que los estudiantes hayan desarrollado las habilidades de aprendizaje necesarias para emprender estudios posteriores con un alto grado de autonomía), la CU2 (Conocer y perfeccionar el nivel de usuario en el ámbito de las TICs), y las competencias específicas de cada una de las asignaturas implicadas (CEC5 conocimiento de los fundamentos de electrónica, CEEI6 Capacidad para diseñar sistemas electrónicos analógicos, digitales y de potencia, y la CEB4 Capacidad para comprender y aplicar los conocimientos básicos de la química general, química orgánica e inorgánica y sus aplicaciones en la ingeniería).

\section{OBjetivos}

Por la modalidad en la que se solicitó este proyecto, el objetivo general que se ha alcanzado es: Introducir a los profesores noveles en la impartición de docencia aplicando un método novedoso e innovador, ya que la interdisciplinariedad de contenidos entre distintas asignaturas será enriquecedor para todos. Se han creado actividades utilizando materiales de gran versatilidad para llevar al aula. Se ha conseguido unir el contenido de asignaturas que están presentes en las tres titulaciones de Graduado en Ingeniería, como son las asignaturas base de tipo general de Química y Fundamentos de Electrónica, pertenecientes a $1^{\circ}$ y $2^{\circ}$ curso de los grados de Ingeniería Eléctrica, Electrónica y Mecánica, así como la asignatura de Diseño 
de circuitos electrónicos y microelectrónicos de $4^{\circ}$ curso del Grado de Ingeniería Electrónica Industrial de la Escuela Politécnica Superior de Córdoba.

Pretendemos que sirva como idea de proyección a otros docentes, ya que al mismo tiempo que se forma un profesor, genera un material útil para que pueda ser consultado por otros compañeros docentes, así como por el alumnado.

Para alcanzar este objetivo general nos basaremos en los siguientes objetivos específicos:

- Coordinación vertical en los grados.

- Optimizar el aprendizaje de contenidos de química

- Aplicar conocimientos de electrónica a sensores utilizados en la asignatura de química

- Contextualizar el proceso de diseño electrónico

- Desarrollar videos didácticos.

- Mejorar el rendimiento académico de los estudiantes

- Mejorar la capacidad de generación de ideas

- Utilizar materiales de lengua inglesa para fomentar el plurilingüismo

- Habilitar una web para depositar los vídeos didácticos

\section{MetodologíA}

Cada una de las partes en las que se divide el proyecto ha demandado diferentes materiales, de acuerdo a la disciplina a tratar.
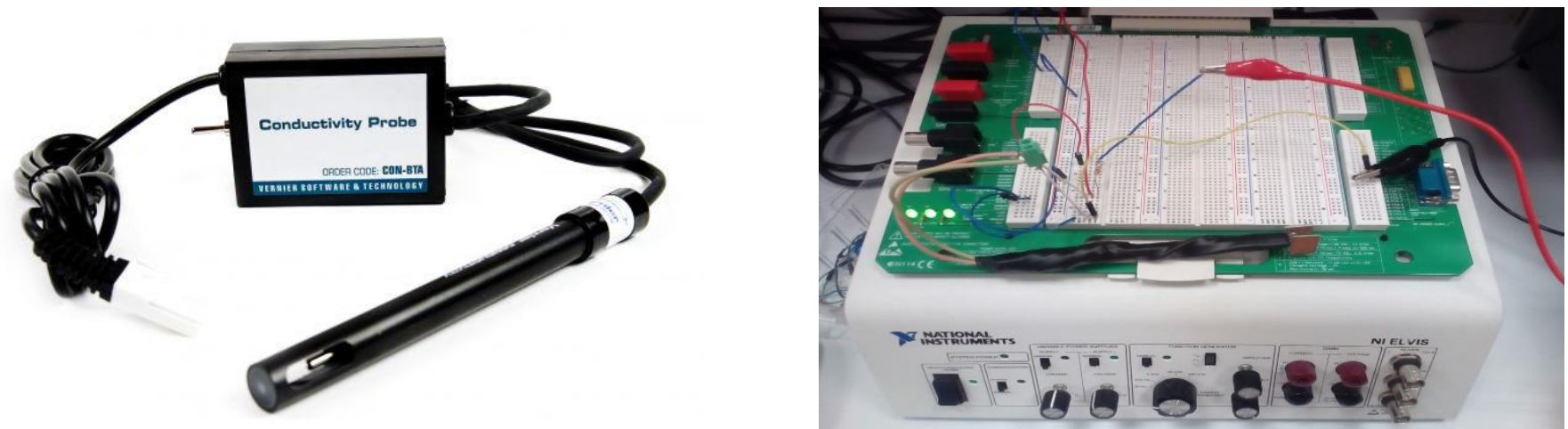

IMAGEN 1. Material empleado para la realización de la medida de conductividad.

Sensor de conductividad Vernier-Ibérica

$>$ Software Logger-Pro

$>$ Plataforma educativa NI ELVIS de National Instrument

$>$ Software Labview

$>$ Cadstar

$>$ Equipos del laboratorio de electrónica (fuente de alimentación, osciloscopio, etc.)

$>$ Equipos del laboratorio de circuitos

La metodología se ha agrupado en tres fases:

\subsection{PRIMERA FASE: Diseño prácticas}

Los profesores noveles, coordinados por sus tutores, han diseñado una serie de prácticas para ser grabadas: como lo visto despierta el interés para conocer sus causas, esto motivará al estudiante para aprender cómo debe estudiarse la ciencia basada en el método científico: observación, toma de datos e interpretación.

La experiencia que hemos diseñado durante el presente curso dentro de las asignaturas implicadas es la siguiente:

- Química: Utilización de un sensor de conductividad para poner de manifiesto la distinta naturaleza de la materia en base al tipo de enlace que existe entre sus átomos. El sensor va conectado a un ordenador y el análisis de los resultados se lleva a cabo con un software que detecta la variable medida por el sensor de conductividad.

- Fundamentos de Electrónica: Parte del contenido de esta asignatura se centra en enseñar a los estudiantes a diseñar y desarrollar distintos tipos de sensores capaces de conectar el mundo físico con el mundo digital. Además, se hace énfasis de la importancia de estos desde el punto de vista ingenieril. Por ejemplo, en las instalaciones de paneles solares, es importante el uso de sensores para medir parámetros como humedad, nivel de radiación, viento, etc. con el fin de optimizar la generación de energía. En esta primera fase, se les propone a los alumnos la tarea de diseñar y desarrollar un sensor de conductividad usando la plataforma de educación NI ELVIS . Esta plataforma, además de permitir un prototipado rápido del sensor, también les ha permitido a los 
alumnos conectar el sensor con el ordenador para graficar los datos obtenidos por este. Una vez desarrollado el sensor, los alumnos tienen que usar líquidos con distintos niveles de conductividad para obtener la curva de calibración del sensor.

- Diseño de Circuitos Electrónicos y Microelectrónicos: Implementación como circuito impreso del sistema seleccionado pasando de la concepción ideal del circuito electrónico a su fabricación real. En dicho proceso se pone de manifiesto la naturaleza concurrente del diseño electrónico ya que el alumno no solo se debe de preocupar del funcionamiento del sistema desde el punto de vista funcional o de componentes, sino que la implementación física lleva consigo el análisis del comportamiento térmico, mecánico y electromágnetico. Del mismo modo, el circuito impreso fabricado deberá tener en cuenta otros aspectos como la fiabilidad, la fabricabilidad y la mantenibilidad. Durante todo este proceso el alumno ha hecho uso de herramientas profesionales de CAD/CAE. Entre todo el abanico de aplicaciones finalmente se ha elegido la plataforma NI Multisim y Ultiboard, si bien, las experiencias están centradas en los aspectos de diseño que deben ser considerados, más que en la implementación concreta de dichos aspectos en la herramienta, de modo que el alumno no se ve limitado a un software concreto, sino que puede aplicar los conceptos a cualquier otra plataforma CAD.

\subsection{SEGUNDA FASE: Elaboración Videos}

En esta fase se han elaborado los vídeos. Estos están pensados como herramienta para que cualquier alumno adquiera los conocimientos previos necesarios como para desarrollar las prácticas de manera autónoma. Los vídeos comienzan con unas cuestiones previas que sirven para conocer el grado de conocimiento previo a la sesión práctica. A continuación se presenta una breve introducción teórica en la que se exponen los conceptos que aparecen en la sesión, así como los cálculos matemáticos, en caso necesario, teniendo una duración aproximada de 5 minutos. Posteriormente, se explican los pasos necesarios para la simulacion y el montaje final.

Incluye el uso de los diversos programas empleados en cada una de las fases (Multisim para la simulación de los cicuitos, NI ELVIS, Ultiboard para el diseño electrónico,..).

Estos videos se encuentran publicados en You Tube. Están grabados en castellano, y subtitulados en inglés, al cual se accede a través del icono situado en la parte inferior derecha de los videos (ver Imagen 2).

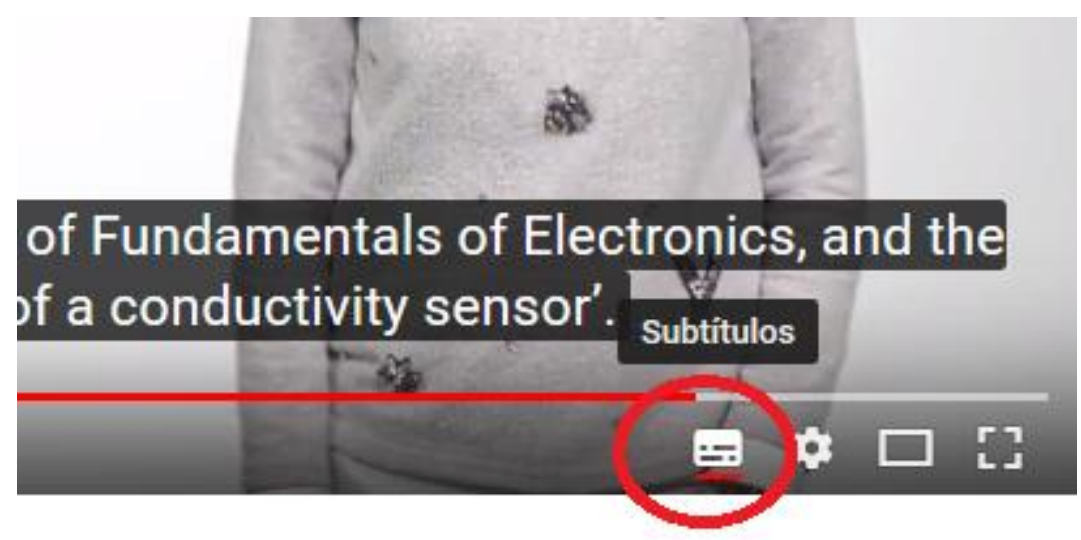

IMAGEN 2. Material empleado para la realización de la medida de conductividad

\subsection{EVALUACION: Web}

Realización de una página web en la que albergar los vídeos de las distintas prácticas. Hoy en día se ofrecen diversas herramientas de creación web. Se ha elegido Sites de Google, al ser la que mejor se adecúa a las características de los videos. Y además, la web está disponible para cualquier profesor o alumno que quiera utilizarla ya que está abierta a todos los usuarios. Se encuentra realizada en castellano e inglés. La dirección web es:

$$
\text { https://sites.google.com/view/en-innovauco?authuser }=0
$$




\section{RESULTADOS OBTENIDOS Y DISCUSIÓN}

Los resultados han sido satisfactorios dado que se presentan actividades relacionadas con la enseñanza/aprendizaje de disciplinas de interés en Ingeniería y que de forma tradicional no se han relacionado.

Los profesores noveles que han participado en este proyecto actualmente imparten las asignaturas para los que va dirigido este proyecto. El material videográfico obtenido permitirá la docencia de conceptos de química y electrónica, transmitiendo a los alumnos la continuación e interdisciplinariedad entre asignaturas de la titulación. Asímismo fomentará la coordinación entre el profesorado, que al pertenecer a distintos departamentos, trabaja conjuntamente en adaptar contenidos para facilitar el aprendizaje del alumnado.

Esperamos que la coordinación de profesores que han llevado a cabo este proyecto sea el inicio para que el alumno tome conciencia que un ingeniero necesita un conocimiento global de las materias que estudia. Dado que el sistema educativo actual está orientado a la evaluación por competencias, y que estas son comunes en distintas asignaturas, una vez que finalice este proyecto, se pretende valorar la posibilidad de generar un sistema de evaluación global en las asignaturas implicadas, de forma que la puntuación le sirva para más de una asignatura.

Una vez ejecutado, se desprende que este proyecto ha servido para sincronizar, implicar y aplicar desarrollo de competencias tanto específicas como transversales a varias asignaturas de las titulaciones de ingeniería. El resultado tiene una elevada componente de plurilingüismo, ya que los vídeos se han sustitulado también al inglés, resultado que favorecerá la comprensión y estímulo del autoaprendizaje en inglés durante la formación de ingeniería.

Los videos se encuentran publicados en una página web de acceso público, además de estar disponibles en YouTube. La página web consta de varias páginas enlazadas:

$>$ página de inicio, donde se describen las principales características del proyecto

$>$ página de videos, donde se albergan 4 vídeos, el $1^{\circ}$ es el de presentación del proyecto, el $2^{\circ}$ corresponde a la primera experiencia (Uso del sensor de conductividad para estudiar aspectos químicos), el $3^{\circ}$ corresponde a la segunda experiencia (Diseño de un sensor de conductividad y los componentes electrónicos necesarios), y el $4^{\circ}$ y último video corresponde a la última experiencia (Diseño del circuito electrónico del sensor de conductividad)

$>$ página de integrantes, donde se describe el profesorado implicado en la realización del proyecto

$>$ recursos, en el que se detallan los materiales y bibliografía usados a lo largo del proyecto

Finalmente, el último icono sirva para cambiar el idioma (Inglés/Castellano). El enlace web para acceder es: https://sites.google.com/view/es-innovauco/inicio

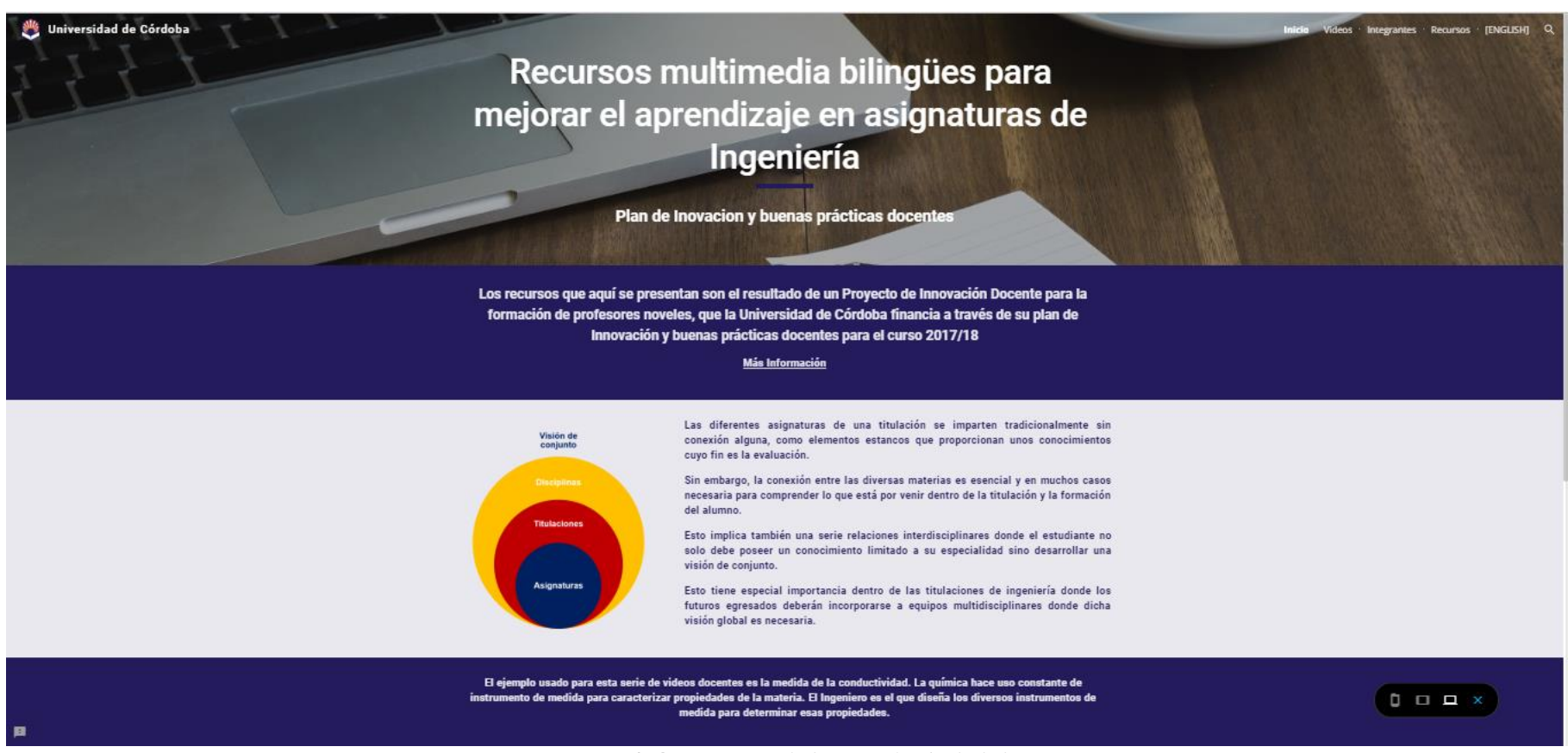

IMAGEN 3. Página web de inicio 


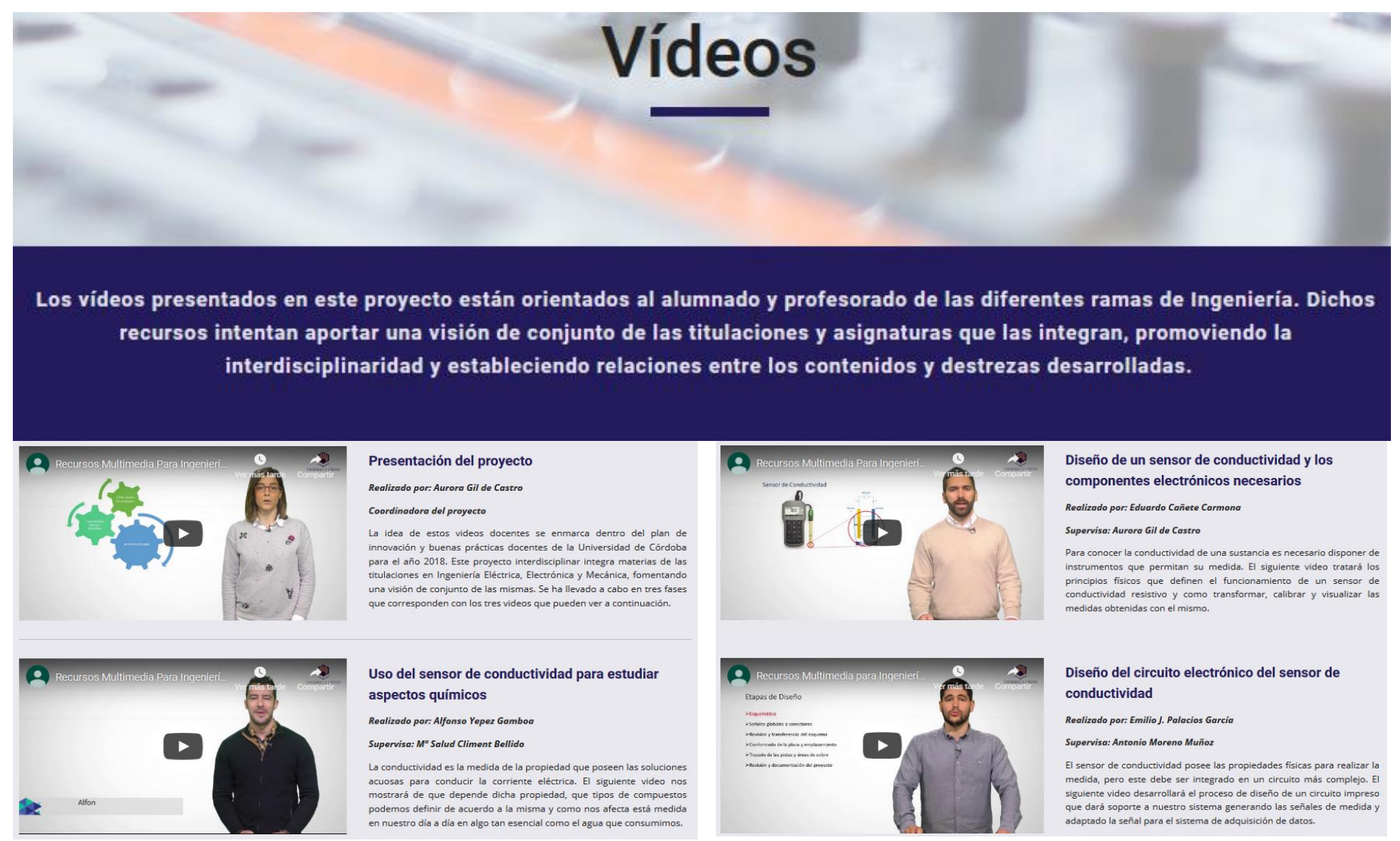

IMAGEN 4. Página web con los videos docentes en castellano.

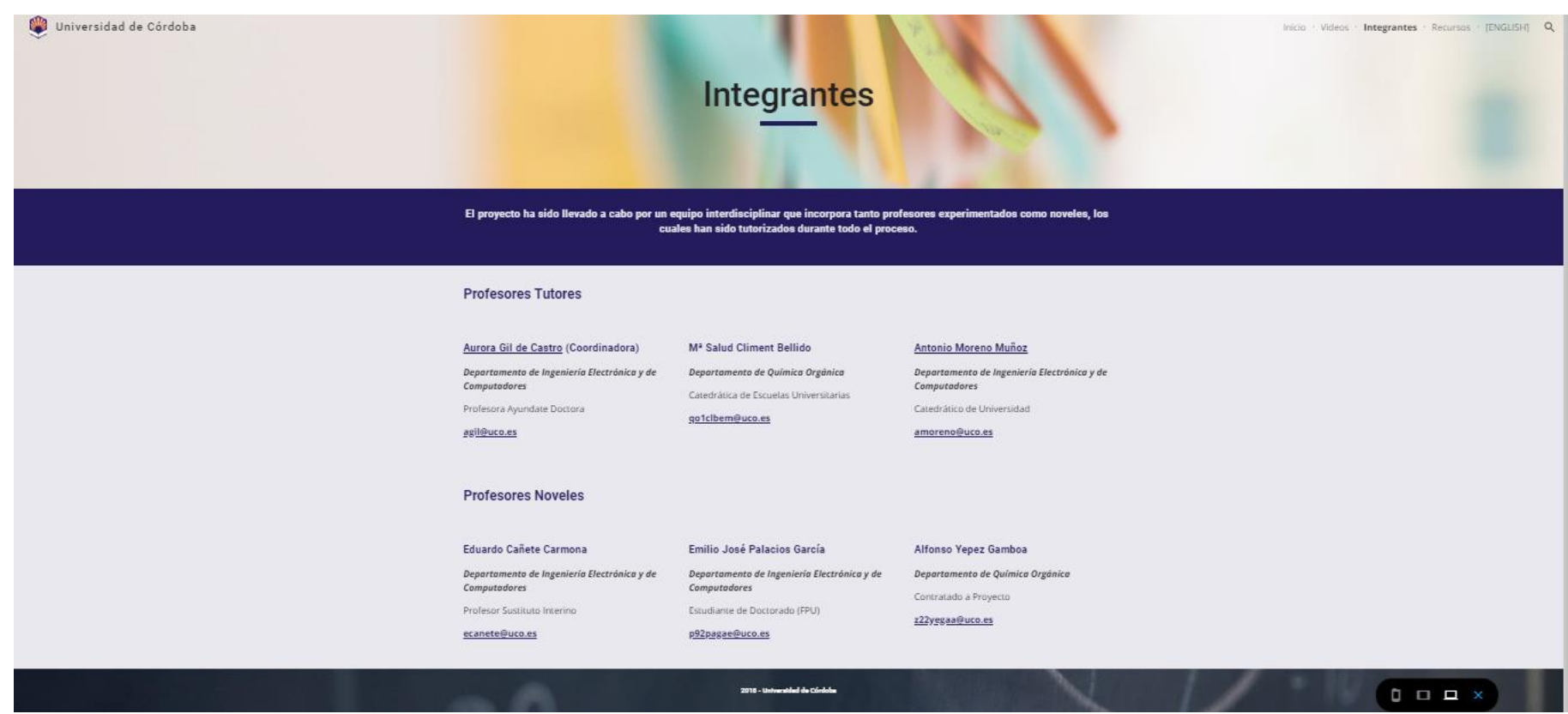

IMAGEN 5. Página web con los integrantes del proyecto 


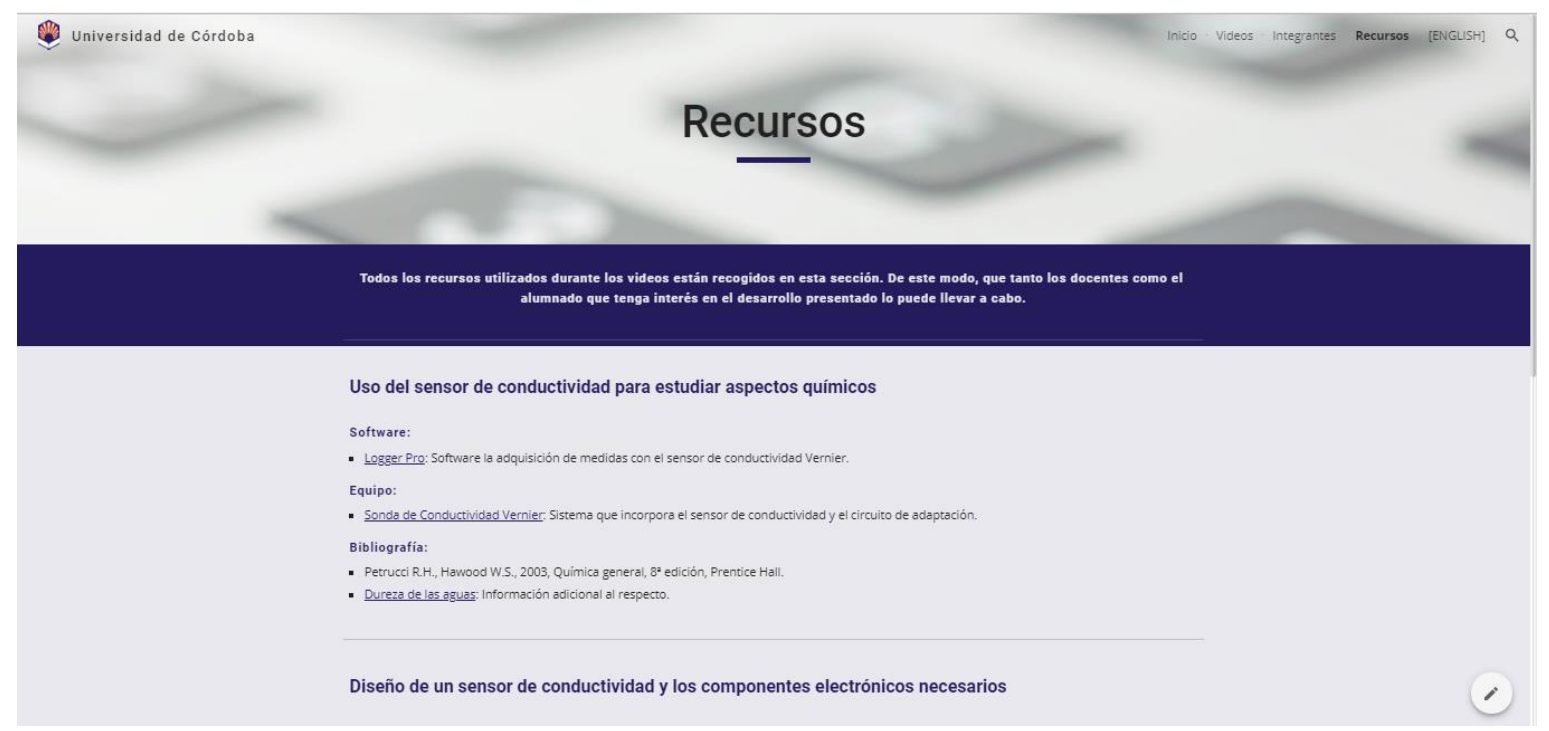

IMAGEN 6. Página web con los recursos utilizados en el proyecto.

\section{CONCLUSIONES}

Con este proyecto se desarrollan varias competencias transversales y específicas de las titulaciones de grado de Ingeniería Industrial. Se presentan actividades relacionadas con la enseñanza/aprendizaje de disciplinas de interés en Ingeniería y que de forma tradicional no se han relacionado. Además, el trabajo desarrollado es interdisciplinar; ya que reúne conceptos de diversas disciplinas, conformando un amplio conocimiento en los futuros ingenieros que cursan los diversos grados, y dándoles una visión de conjunto de la titulación desde el primer curso.

Con los recursos multimedia bilingües presentados en esta memoria se podrían realizar aquellas prácticas que por su duración y coste de los elementos empleados no se podrían implementar a modo particular con todo el alumnado. Aprovechar el uso de las nuevas tecnologías como el aprendizaje multimedia resulta esencial, más aún si se realiza en un segundo idioma, para su formación como ingenieros.

Estos recursos también pueden suponer mejoras a nivel de título, ya que el poner de manifiesto el nexo de unión entre varias asignaturas ayuda al aprendizaje, además de ofrecer una prospección de futuro en las diversas asignaturas del grado, lo que motivará y alentará al alumnado en promocionar a los siguientes cursos.

\section{AGRADECIMIENTOS}

El trabajo ha sido financiado por el Plan de Innovación y Buenas Prácticas Docentes 2017-2018, en concreto en la modalidad 2, Proyectos de Innovación para Formación en Innovación Docente..

\section{BIBLIOGRAFÍA}

1. Espacio Europeo de Educación Superior, http://www.eees.es/, acceso 07/06/2018.

2. ARISTIMUÑO, A., "Las competencias en la educación superior: ¿demonio u oportunidad?”, III Congreso Internacional de Docencia Universitaria e Innovación, Gerona, pp. 3-8, Junio 2009.

3. WALSH K, RAFIQ I, HALL R. "Online educational tools developed by Heart improve the knowledge and skills of hospital doctors in cardiology". Postgrad Med J, 83:502-503. 2007

4. Nilsson M, Bolinder G, Held C, Johansson BL, Fors U, Östergren J. "Evaluation of a web-based ECGinterpretation programme for undergraduate medical students". BMC Medical Education, 8: 25. 2008

5. National Instrument (NI) ELVIS, http://www.ni.com/ni-elvis/what-is/esa/, acceso 07/06/2018

6. National Instrument (NI) Ultiboard, http://sine.ni.com/np/app/main/p/docid/nav-98/lang/es/fmid/10811/, acceso $07 / 06 / 2018$ 